\title{
SOLUTION OF PLANE DIRICHLET PROBLEM USING COMPACTLY SUPPORTED 2D WAVELET SCALING FUNCTIONS
}

\author{
Andrzej Katunin \\ Department of Fundamentals of Machinery Design, Silesian University of Technology, Poland \\ andrzej.katunin@polsl.pl
}

\begin{abstract}
The paper presents the solution of the homogeneous plane Dirichlet problem using the wavelet-Galerkin method with various $2 \mathrm{D}$ compactly supported wavelet scaling functions. An analysis of approximation accuracy was performed with respect to the orders of investigated wavelet scaling functions and the level of approximation. The most effective scaling functions for solving the Dirichlet problem were indicated and discussed.
\end{abstract}

\section{Introduction}

The wavelets theory is relatively new, but an effective mathematical tool for solving many theoretical and engineering problems. The wide range of its applications covers developed numerical algorithms for approximation of ordinary differential equations (ODE) and partial differential equations (PDE), image processing, signal analysis etc. The algorithms based on wavelet theory for solving ODE and PDE offer several advantages in comparison with the algorithms based on finite difference and finite element methods, e.g. low time-consumption and good accuracy [1].

The two most popular wavelet-based methods for solving differential equations (DE) are [2]: the collocation method and the wavelet-Galerkin method (WGM). According to some good properties, e.g. the possibility of multiresolution analysis and stability of decomposition and reconstruction in spaces of square-integrable functions $L^{2}\left(\mathbf{R}^{\mathrm{n}}\right)$, solution stability, WGM gain great popularity.

The crucial task in application of wavelet-based numerical approximation algorithms is the choice of appropriate wavelet scaling functions. In the function approximation problems, the wavelets with infinite support results in poor effects, therefore the orthogonal wavelets were primarily chosen for solving DE [3]. One of the most popular wavelets is the Daubechies' $(d b)$ wavelet, which is often used for solving DE $[4,5]$. However, several authors used other wavelet scaling functions for solving DE, e.g. symlets (sym), coiflets (coif), B-spline wavelets ( $b s p)$, biorthogonal (bior) and reversed biorthogonal (rbio) wavelets [6-8].

Due to the separability property of the above-mentioned compactly supported wavelets, they could be simply generalized to the higher dimensions by tensor 
product of wavelet and scaling functions. Thus, WGM could be adapted to the 2D and $\mathrm{nD}$ problems. Some solutions of multi-dimensional problems with use of WGM were already presented. The authors of [9] present the solution of 2D Dirichlet problem using 2D Daubechies wavelet scaling functions. The same scaling functions were used for the 2D steady electro-magnetic problem [10] and 2D heat equations [11]. The 2D problems of mechanical elasticity [12] and non-steady diffusion [13] were solved with use of the 2D B-spline wavelet scaling functions.

In the present study the plane Dirichlet problem with homogeneous boundary conditions was solved using all known discrete compactly supported wavelet scaling functions with various orders. Firstly, the influence of type and order of the scaling functions applied to the problem on approximation accuracy was investigated and discussed. Then, for the chosen wavelet scaling functions, which give the most accurate approximation results, the problem was solved on various levels of approximation for estimating the character of error decay.

\section{Wavelet analysis and compactly supported wavelets}

One of the most useful properties of wavelet-based methods is the multiresolution analysis (MRA) of functions introduced by Mallat [14]. Thanks to the consideration of existence of orthonormal (semi- or biorthogonal) Riesz bases in $L^{2}\left(\mathbf{R}^{\mathrm{n}}\right)$ MRA provide decomposition and reconstruction stability of the approximated function in different resolutions. The definition of MRA is based on sequential representation of closed functional spaces $V_{j} \subset L^{2}\left(\mathbf{R}^{n}\right)$ :

$$
\ldots V_{2} \subset V_{1} \subset V_{0} \subset V_{-1} \subset V_{-2} \ldots
$$

with properties

$$
\bigcup_{j \in \mathbf{Z}} V_{j}=L^{2}\left(\mathbf{R}^{n}\right), \bigcap_{j \in \mathbf{Z}} V_{j}=\{0\}
$$

In the $1 \mathrm{D}$ case the scaling functions of wavelets are constructed based on the twoscale relation:

$$
\varphi(x)=\sum_{k} p_{k} \varphi(2 x-k)
$$

where $\left\{p_{k}\right\}$ is a finite set of two-scale coefficients, which determines the compact support for $p_{k} \neq 0$. The Riesz basis $\left\{\varphi_{j, k}(x)\right\}$ for a given subspace $V_{j} \subset L^{2}(\mathbf{R})$ determines translations and dilations of scaling functions on the level $j$ as:

$$
\varphi_{j, k}(x)=2^{j / 2} \varphi\left(2^{j} x-k\right), k \in \mathbf{Z} .
$$


The multi-scale relation (4) could be generalized to $\mathbf{R}^{2}$ as follows:

$$
\varphi_{j, k, l}(x, y)=2^{j / 2} \varphi\left(2^{j} x-k, 2^{j} y-l\right), k, l \in \mathbf{Z} .
$$

Considering multi-scale relation (5), which constitutes the orthonormal base in space $V_{j}$ the tensor product scaling function in $\mathbf{R}^{2}$ takes a form:

$$
\Phi(x, y)=\varphi(x) \otimes \varphi(y) .
$$

All of the compactly supported wavelets mentioned above match two-scale relation (5), but each of them has specific properties, which have an influence on their approximation effectiveness. The family of $d b$ wavelets [15] is characterized by orthogonality, but their scaling functions are not symmetric and have a support width of $[0,2 m-1]$, where $m$ is an order of wavelet. The family of sym wavelets, developed also by Daubechies [15], is a modification of $d b$ wavelets with increased symmetry. The scaling functions of these wavelets are not strictly symmetric; the support width is the same as for $d b$ wavelets. The coif wavelets were developed by Daubechies at the request of Coifman. The scaling functions of these wavelets are characterized by relatively large width of support: $[0,6 m-1]$ and $2 m-1$ vanishing moments equal to 0 [15]. The $b s p$ wavelet scaling functions have the support width of $[0, m]$, are symmetric and orthogonal [16]. The last two families, bior and rbio wavelets, use two different functions for decomposition and reconstruction, thus the decomposition and reconstruction bases are not orthogonal. But in the single base two functions of decomposition and reconstruction are orthogonal. The decomposition scaling function of bior is the same as reconstruction scaling function of rbio and vice versa. The scaling functions of bior and rbio have the support width of $[0,2 m-1]$, where $m$ is the order of decomposition function, and are symmetric [15]. The exemplary tensor product wavelet scaling functions of different wavelet families were presented in Figure 1.

\section{Wavelet-Galerkin method and problem formulation}

Consider the Dirichlet problem in a bounded domain $\Omega$ in $\mathbf{R}^{2}$ with boundary $\Gamma$ :

$$
\begin{gathered}
-\alpha \Delta u+u=f, \\
u=g \text { on } \Gamma .
\end{gathered}
$$

where $\alpha$ is some positive constant. In some cases the explicit solution of (7) does not exist, thus the problem should be reformulated in terms of variational formulation as follows [9]:

$$
u \in V_{g}^{1}: \mathcal{A}(u, v)=f v \mathrm{~d} x \mathrm{~d} y, \forall v \in V,
$$




$$
\mathcal{A}(u, v)=\int_{\Omega}(\alpha \nabla u \nabla v+u v) \mathrm{d} x \mathrm{~d} y,
$$

where $\mathcal{A}(u, v)$ is a bilinear form in the Sobolev space $\mathrm{H}^{1}$ and

$$
V=\left\{v \in \mathrm{H}^{1}(\Omega): v=0 \text { on } \Gamma\right\}, V_{g}=\left\{v \in \mathrm{H}^{1}(\Omega): v=g \text { on } \Gamma\right\} .
$$
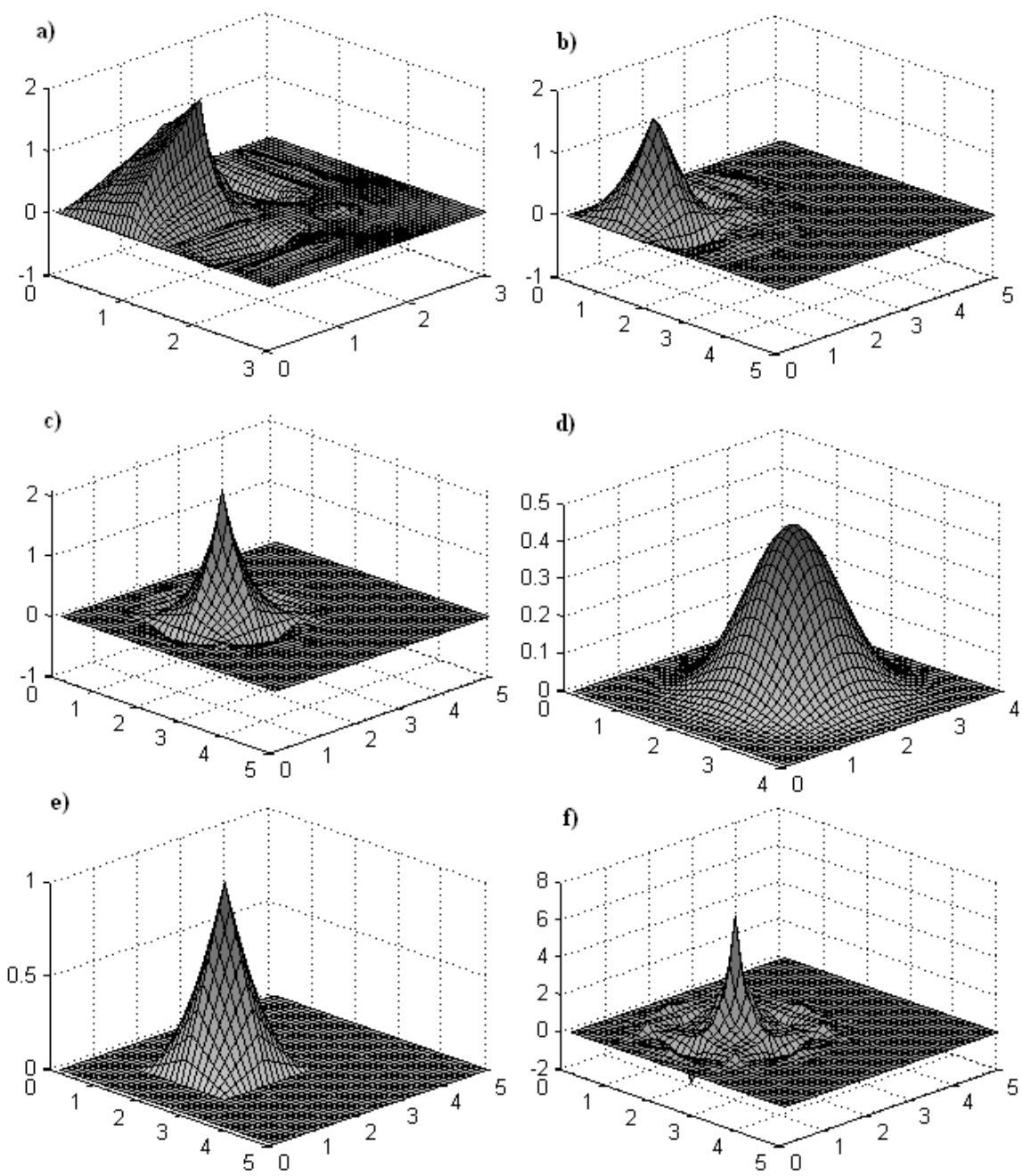

Fig. 1. 2D scaling functions of: a) $d b 2$, b) sym3, c) coif1, d) bsp4, e) bior 2.2 , f) rbio2.2

Considering that (8) has unique solution the wavelet-Galerkin spaces on the approximation level $j$ could be introduced with respect of Lax-Milgram theorem and MRA: 


$$
V_{j}(0, g):=\left\{v \in L^{2}(0, g): v(x)=\sum_{k \in \mathbf{Z}} c_{k} \varphi_{k}^{j}(x), x \in[0, g]\right\},
$$

where $c_{k}=c_{k+n_{j}}, n_{j}$ is a dimension of $V_{j}$. The variational formulation (8) could be expanded for arbitrary $j$ as:

$$
\mathcal{A}\left(u^{j}, v^{j}\right)=f v^{j} \mathrm{~d} x \mathrm{~d} y, \forall v^{j} \in V_{j} \otimes V_{j} .
$$

Considering that

$$
u^{j}(x, y)=\sum_{k, l \in Z} u_{k, l}^{j} \varphi_{k}^{j} \varphi_{l}^{j}, f_{j}^{k, l}=\int_{\Omega} f(x, y) \varphi_{k}^{j} \varphi_{l}^{j} \mathrm{~d} x \mathrm{~d} y
$$

The Galerkin approximation could be performed by solving the following linear system [9]:

$$
\alpha\left(c_{j} u^{j}+u^{j} c_{j}\right)+u^{j}=f^{j}
$$

where $c_{j}$ is a circulant square matrix and $f^{j}$ is a vector with dimensions of $n$.

For solving (14) the derivatives of $f(x)$ in terms of $\varphi(x)$ should be determined. The WG approximation for a derivative of $f(x)$ could be presented as:

$$
f^{(d)}=\sum_{k} p_{k} \varphi_{k}^{(d)}(x),
$$

where $(d)$ denotes differentiation. The derivatives $\varphi_{k}^{(d)}(x)$ could be approximated as

$$
\varphi_{k}^{(d)}=\sum_{s} \lambda_{s} \varphi_{s}(x)
$$

where

$$
\lambda_{s}=\int_{-\infty}^{\infty} \varphi_{k}^{(d)}(x) \varphi_{s}(x) \mathrm{d} x .
$$

The set of connection coefficients $\lambda_{s}$ could be obtained for a chosen wavelet scaling function from a universal formula for compactly supported proposed by Latto et al. in [17]:

$$
\Lambda_{k_{1}, k_{2}, \ldots, k_{n}}^{\left(d_{1}\right),\left(d_{2}\right), \ldots\left(d_{n}\right)}=\int_{-\infty}^{\infty} \prod_{i=1}^{n} \varphi_{k}^{(d)}(x) \mathrm{d} x .
$$

Following to the investigated problem (7) the two-term connection coefficients for all of considered wavelets should be calculated as: 


$$
\Lambda_{k}^{\left(d_{1}\right),\left(d_{2}\right)}=\int_{-\infty}^{\infty} \varphi^{\left(d_{1}\right)}(x) \varphi_{k}^{\left(d_{2}\right)}(x) \mathrm{d} x
$$

\section{Numerical example and analysis of results}

Let us consider (7) with homogeneous Dirichlet boundary conditions, i.e.: $V=V_{g}=\mathrm{H}_{0}^{1}(\Omega), g(\Omega) \equiv 0$ and $\alpha(\Omega) \equiv 0$. The problem was solved using fictitious domain formulation developed by Glowinski et al. [18]. Assume that the problem domain $\Omega$ and fictitious domain $\Xi$ are rectangular. The problem could be presented as a dyadic MRA-based construction $h=2^{j}$ for a finite dimensional space $V_{j}$. Considering the fictitious approach the problem (7) could be formulated as follows:

$$
\int_{\Xi} \nabla u_{\varepsilon} \nabla v+\varepsilon^{-1} \int_{\partial \Omega} u_{\varepsilon} v=\int_{\Xi} \tilde{f} v
$$

where $\varepsilon$ is a positive penalty parameter and $\widetilde{f}$ is the $L^{2}$-extension of $f$ to $\Xi$. Considering (20) the linear system (14) takes form:

$$
c_{j} u^{j}+\varepsilon^{-1} M_{j} u^{j}=f^{j},
$$

where $M_{j}$ is the boundary integral for the approximation level $j$ given by [17]:

$$
M_{i}^{j}=\int_{-\infty}^{\infty} x^{j} \varphi(x) \mathrm{d} x .
$$

For numerical example $f$ in (21) was defined as:

$$
f(x, y)=\sin \pi x \sin \pi y .
$$

\subsection{Estimation of approximation errors}

The problem solving was carried out using a numerical algorithm implemented following WGM with fictitious domain approach as a hybrid MATLAB ${ }^{\circledR} / \mathrm{C}$ code. The domains were defined as follows: $\Omega=[1,2] \times[1,2], \Xi=[0,3] \times[0,3]$. The calculations were performed using $d b, s y m, b s p$, coif wavelet scaling functions with order started from 2 and bior and rbio primal wavelet scaling functions with order from 1 for $j=4$. For every computed case, three parameters were estimated: absolute error distribution

$$
e^{j}=\left|\widetilde{u}^{j}(x, y)-u^{j}(x, y)\right|,
$$


where $\tilde{u}^{j}$ denotes exact solution, maximal value of absolute error distribution $\max \left(e^{j}\right)$ and maximal values absolute error

$$
e_{\max }^{j}=\left|\max \left(\tilde{u}^{j}(x, y)\right)-\max \left(u^{j}(x, y)\right)\right| .
$$

The values of $\max \left(e^{j}\right)$ and $e_{\max }^{j}$ for cases, which have $e^{4}<0.1$ were tabulated in Table 1. Exemplary error distributions were presented in Figure 2.

Table 1

Errors of solution using various wavelet scaling functions

\begin{tabular}{|c|c|c|c|c|c|}
\hline Wavelet & $d b 3$ & $d b 4$ & $d b 5$ & $d b 6$ & $d b 8$ \\
\hline $\max \left(e^{j}\right)$ & $3.84433 \cdot 10^{-2}$ & $1.23923 \cdot 10^{-2}$ & $3.83776 \cdot 10^{-2}$ & $8.57375 \cdot 10^{-2}$ & $7.36402 \cdot 10^{-2}$ \\
\hline$e_{\max }^{j}$ & $1.43353 \cdot 10^{-3}$ & $1.60384 \cdot 10^{-4}$ & $3.61948 \cdot 10^{-3}$ & $2.00560 \cdot 10^{-3}$ & $5.35092 \cdot 10^{-2}$ \\
\hline \hline Wavelet & $d b 9$ & $d b 10$ & $s y m 3$ & coif 2 & $b s p 2$ \\
\hline $\max \left(e^{j}\right)$ & $4.80419 \cdot 10^{-2}$ & $3.27045 \cdot 10^{-2}$ & $3.80749 \cdot 10^{-2}$ & $9.92343 \cdot 10^{-2}$ & $7.38519 \cdot 10^{-3}$ \\
\hline$e_{\max }^{j}$ & $4.73401 \cdot 10^{-2}$ & $1.86240 \cdot 10^{-3}$ & $3.72645 \cdot 10^{-3}$ & $9.22704 \cdot 10^{-2}$ & $7.18076 \cdot 10^{-3}$ \\
\hline \hline Wavelet & $b s p 4$ & $b s p 5$ & $b s p 6$ & $b s p 7$ & bior 2.2 \\
\hline $\max \left(e^{j}\right)$ & $1.77807 \cdot 10^{-2}$ & $8.89205 \cdot 10^{-2}$ & $3.41012 \cdot 10^{-2}$ & $8.79539 \cdot 10^{-2}$ & $1.06277 \cdot 10^{-2}$ \\
\hline$e_{\max }^{j}$ & $1.28696 \cdot 10^{-2}$ & $2.40170 \cdot 10^{-2}$ & $1.89351 \cdot 10^{-2}$ & $1.20829 \cdot 10^{-2}$ & $1.04361 \cdot 10^{-2}$ \\
\hline
\end{tabular}
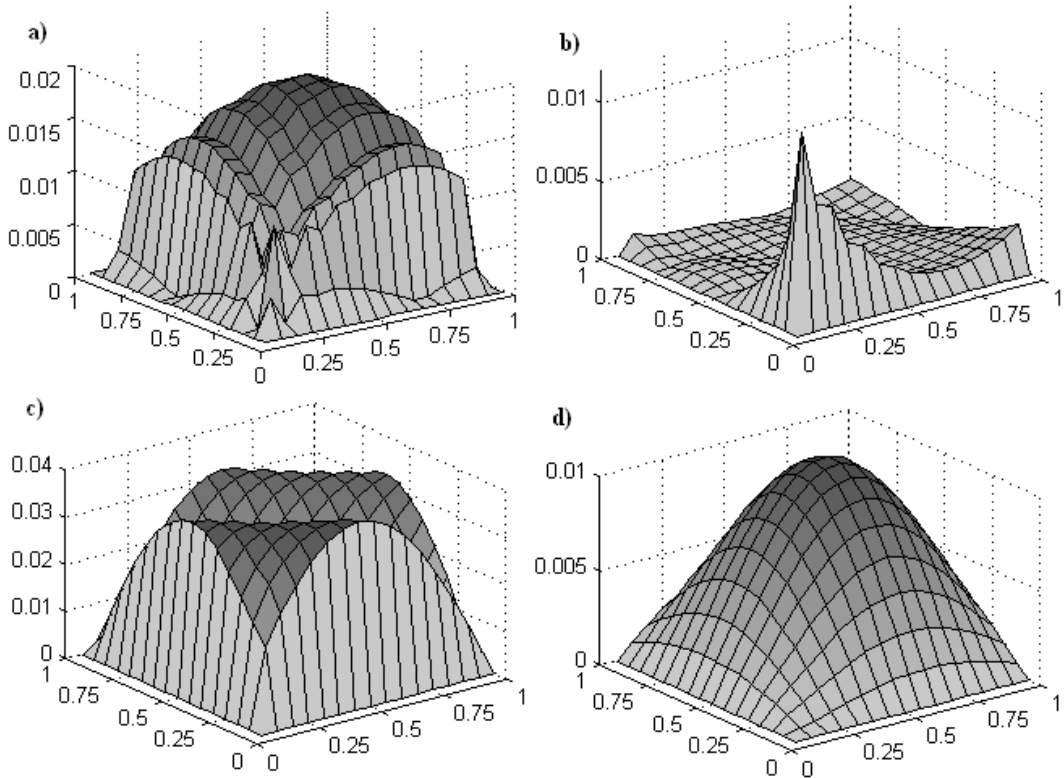

Fig. 2. Error distributions for: a) $b s p 4$, b) $d b 4$, c) $s y m 3$, d) bior 2.2 for $j=4$ 
Results presented in Table 1 show, that the most suitable wavelet families for approximation are $d b$ and $b s p$, because most of them provide accurate results with an error below the threshold defined above. Error distribution in each particular case of applied wavelet scaling function has a different character, which could be noticed in Figure 2. An error distribution is mainly determined by connection coefficients, which are unique for every type and every order of wavelet scaling function.

\subsection{Influence of approximation level on the solution accuracy}

Following the results presented in Table 1 the most effective wavelet scaling functions $(d b 4, b s p 2, b s p 4$ and bior2.2) were chosen for further calculations. The formulated problem was solved in various approximation levels $j=4 \div 8$ in order to determine the character of an error decay with increase of the approximation level. The computational code was optimized for parallel computing. Obtained results were presented in Figure 3 and in Table 2.

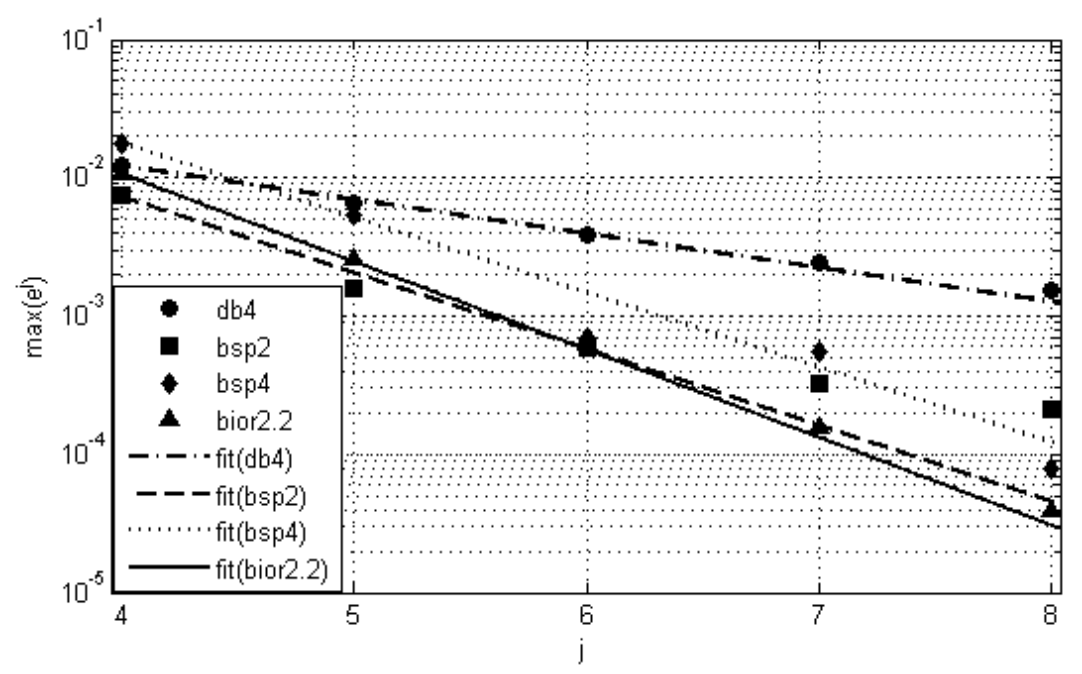

Fig. 3. Maximal values of absolute error distributions for chosen wavelets with respect of level of approximation and fittings of them

It could be observed that $\max \left(e^{j}\right)$ for investigated wavelet scaling functions has a near exponential character. An optimal choice of wavelet for solving DE depends on several parameters. Firstly, an appropriate wavelet scaling function should be chosen while taking into consideration the shape of the approximated function. In the presented problem the most accurate solutions were obtained using symmetric and smooth wavelet scaling functions, however the situation may differ in the case of approximation of more irregular function. 
Table 2

Maximal values of absolute error of solution on various approximation levels

\begin{tabular}{|c|c|c|c|c|c|}
\hline Level & 4 & 5 & 6 & 7 & 8 \\
\hline$d b 4$ & $1.23923 \cdot 10^{-2}$ & $6.51467 \cdot 10^{-3}$ & $3.93259 \cdot 10^{-3}$ & $2.44901 \cdot 10^{-3}$ & $1.53764 \cdot 10^{-3}$ \\
\hline$b s p 2$ & $7.38519 \cdot 10^{-3}$ & $1.60719 \cdot 10^{-3}$ & $5.93929 \cdot 10^{-4}$ & $3.30814 \cdot 10^{-4}$ & $7.97718 \cdot 10^{-5}$ \\
\hline$b s p 4$ & $1.77807 \cdot 10^{-2}$ & $5.33456 \cdot 10^{-3}$ & $6.90199 \cdot 10^{-4}$ & $5.52537 \cdot 10^{-4}$ & $2.14155 \cdot 10^{-4}$ \\
\hline bior 2.2 & $1.06277 \cdot 10^{-2}$ & $2.57899 \cdot 10^{-3}$ & $6.32637 \cdot 10^{-4}$ & $1.55663 \cdot 10^{-4}$ & $3.98178 \cdot 10^{-5}$ \\
\hline
\end{tabular}

The most accurate results were obtained using 2D B-spline wavelet scaling functions and biorthogonal scaling functions. It should be mentioned, that 2D B-spline wavelets applied to damage identification problem also reveal the best results in comparison with other compactly supported wavelets [19]. The second important parameter is the order of the wavelet scaling function. In the case of $d b$ wavelet scaling functions there is no dependence of approximation errors on the order of scaling function. For $b s p$ wavelet scaling functions there is a tendency: odd scaling functions are characterized by lower errors. In the case of other wavelet scaling functions considered in this study errors increased with an increase of the order. Moreover, the order influences on the number of considered connection coefficients and thus, on CPU time. Therefore it is suitable to choose wavelet scaling functions of low order for obtaining the most accurate results in the shortest time.

\section{Conclusions}

The plane Dirichlet problem was solved using wavelet-Galerkin method and fictitious domain formulation with use of various $2 \mathrm{D}$ compactly supported wavelet scaling functions. During performed comparative study of approximation accuracy using above-mentioned scaling functions the best of them were chosen. The aspects of optimal choice of 2D wavelet scaling functions for solving differential equations were presented and discussed.

\section{Acknowledgements}

The author acknowledges a possibility of carrying out computations on the IBM BladeCenter HS21 in Academic Computer Centre CYFRONET AGH under the computational grant No. MNiSW/IBM BC HS21/PŚlaska/047/2011. 


\section{References}

[1] Chui C.K., Wavelets: A Mathematical Tool for Signal Analysis, SIAM Monographs on Mathematical Modeling and Computation, Society for Industrial and Applied Mathematics, Pennsylvania 1997.

[2] Nikolaou M., You Y., Use of Wavelets for Numerical Solution of Differential Equations, [in:] Wavelet Applications in Chemical Engineering, eds. B. Joseph, R. Motard, Kluwer 1994, 209-274.

[3] Lakestani M., Razzaghi M., Dehghan M., Solution of nonlinear Fredholm-Hammerstein integral equations by using semiorthogonal spline wavelets, Math. Probl. Eng. 2005, 1, 113-121.

[4] Neelov A.I., Goedecker S., An efficient numerical quadrature for the calculation of potential energy of wavefunctions expressed in the Daubechies wavelet basis, J. Comput. Phys. 2006, 217 , 312-339.

[5] Liu Ya, Liu Yi, Cen Zh, Multi-scale Daubechies wavelet-based method for 2-D elastic problems, Finite Elem. Anal. Des. 2011, 47, 334-341.

[6] Akbari H., Kerayechian A., Coiflet-Galerkin method for solving second order BVPs with variable coefficients in three dimensions, Numer. Algor., in press, doi: 10.1007/s1 1075-012-9558-x.

[7] Nowak Ł.D., Pasławska-Południak M., Twardowska K., On the convergence of the waveletGalerkin method for nonlinear filtering, Int. J. Math. Comput. Sci. 2010, 20, 93-108.

[8] Lakestani M., Dehghan M., The solution of second-order nonlinear differential equation with Neumann boundary conditions using semi-orthogonal B-spline wavelets, Int. J. Comput. Math. 2006, 83, 685-694.

[9] Glowinski R., Rieder A., Wells R.O., Zhou X., A wavelet multigrid preconditioner for Dirichlet boundary-value problems in general domains, Math. Mod. Numer. Anal. 1996, 30, 711-729.

[10] Shiyou Y., Guangzheng N., Jingen Q., Ronglin L., Wavelet-Galerkin method for computations of electromagnetic fields, IEEE Trans. Magn. 1998, 34, 2493-2496.

[11] Hashish H., Behiry S.H., Elsaid A., Solving the 2-D heat equations using wavelet-Galerkin method with variable time step, App. Math. Comput. 2009, 213, 209-215.

[12] Yanan L., Liang S., Yinghua L., Zhangzhi C., Multi-scale B-spline method for 2-D elastic problems, App. Math. Mod. 2011, 35, 3685-3697.

[13] Xiong L., Li H., Zhang L., Two dimensional tensor product B-spline wavelet scaling functions for the solution of two-dimensional unsteady diffusion equations, J. Ocean Univ. Chin. 2008, 7 , 258-262.

[14] Mallat S., A theory of multiresolution signal decomposition: the wavelet representation, IEEE Trans. Pattern Anal. Machine Intell. 1989, 11, 674-693.

[15] Daubechies I., Ten Lectures on Wavelets, SIAM, Philadelphia 1992.

[16] Chui C.K., Introduction to Wavelets, Academic Press Professional, San Diego 1994.

[17] Latto A., Resnikoff H.L., Tenenbaum E., The evaluation of connection coefficients of compactly supported wavelets, Proceedings of the French-USA Workshop on Wavelets and Turbulence, ed. Y. Maday, New York 1992.

[18] Glowinski R., Pan T.W., Wells R.O., Zhou X., Wavelet and finite element solutions for the Neumann problem using fictitious domains, J. Comput. Phys. 1996, 126, 40-51.

[19] Katunin A., Damage identification in composite plates using two-dimensional B-spline wavelets, Mech. Syst. Signal Pr. 2011, 25, 3153-3167. 